\title{
ASSOCIATED TRANSFORMS FOR SOLUTION OF NONLINEAR EQUATIONS
}

\section{JOYATI DEBNATH and NARAYAN C. DEBNATH}

\author{
Department of Mathematics and Computer Science \\ University of Wisconsin \\ River Falls, WI 54022 U.S.A.
}

\begin{abstract}
Nonlinear multivariable differential or integrodifferential equations with terms of mixed dimensionality can be solved using multidimensional Laplace transform. The special technique used to find the inverse of the multidimensional Laplace transform is known as the association of variables. In this paper, some basic theorems are developed for the theory of association. Examples are presented for each theorem. Once the basic theorems are established, it is possible to derive many useful associated pairs.
\end{abstract}

KEY WORDS AND PHRASES. Multidimensional Laplace transform and Nonlinear equations. 1980 AMS SUBJECT CLASSIFICATION CODE. 44A10, 44A30.

\section{INTRODUCTION.}

In systems engineering, nonlinear differential or integrodifferential equations are solved using multiple dimensional Laplace transform. A commonly used method for obtaining the inverse of the multidimensional Laplace transform is called the association of variables. Suppose $F\left(s_{1}, s_{2}, \ldots, s_{n}\right)$ be a Laplace transform. Its $n$-dimensional inverse can be found by the integral

$$
\begin{aligned}
f\left(t_{1}, t_{2}, \ldots, t_{n}\right) \equiv & L_{n}^{-1}\left[F\left(s_{1}, s_{2}, \ldots, s_{n}\right) ; t_{1}, t_{2}, \ldots, t_{n}\right] \\
= & \frac{1}{(2 \pi i)^{n}} \int_{\alpha_{1}-i \infty}^{\alpha_{1}+i \infty} \ldots \int_{\alpha_{n}-i \infty}^{\alpha_{n}+i \infty} \exp \left(\sum_{j=1}^{n} s_{j} t_{j}\right) \\
& \cdot F\left(s_{1}, s_{2}, \ldots, s_{n}\right) \prod_{j=1}^{n} d s_{j}
\end{aligned}
$$

In certain nonlinear systems analysis, particularly in Volterra series applications [1-2] on Nonlinear systems [3-5], it becomes necessary to take the inverse of the $n$-dimensional Laplace transform and specify this inverse image in the special case: $t_{1}=t_{2}=\cdots=t_{n}=t$. We denote this image function of one variable by $g(t)$, or

$$
g(t)=\left.f\left(t_{1}, t_{2}, \ldots, t_{n}\right)\right|_{t_{1}=t_{2}=\cdots=t_{n}=t}
$$

An alternative approach to obtain the time function, $g(t)$, is to associate with given $F\left(s_{1}, s_{2}, \ldots, s_{n}\right)$ a function $G(s)$ from which a direct application of the one- dimensional inverse transform yields $g(t)$. This special method of computing the inverse transform is said to be the association of variables. The function $G(s)$ is called the associated transform of $F\left(s_{1}, s_{2}, \ldots, s_{n}\right)$. 
Recently, Chen and Chiu [6] and Koh [7] have presented several theorems for evaluating the associated transform $G(s)$ using certain types of $F\left(s_{1}, s_{2}, \ldots, s_{n}\right)$. In this paper, a set of new and important theorems are developed. Several illustrative examples are included. However, once the fundamental theorems are established, we can derive many useful associated pairs and use them conveniently.

\section{THEOREMS ON ASSOCIATED TRANSFORMS.}

Suppose $G(s)$ be the associated transform of $F\left(s_{1}, s_{2}, \ldots, s_{n}\right)$ and $G_{1}(s)$ be that of $F\left(s_{1}, \ldots, s_{m-1}, s_{m+1}, \ldots, s_{n}\right), m \leq n$. Let $k$ be any constant and we restrict the variables $s, s_{1}, s_{2}, \ldots, s_{n}$ to the right half of the complex plane.

Theorem 2.1. If a given function $F\left(s_{1}, s_{2}, \ldots, s_{n}\right)$ can be written in the form

$$
F\left(s_{1}, s_{2}, \ldots, s_{n}\right)=\frac{k}{s_{m}\left(s_{m}^{2}+\alpha^{2}\right)} F_{1}\left(s_{1}, \ldots, s_{m-1}, s_{m+1}, \ldots, s_{n}\right)
$$

and if $F_{1}\left(s_{1}, \ldots, s_{m-1}, s_{m+1}, \ldots, s_{n}\right) \stackrel{A_{n-1}}{\longrightarrow} G_{1}(s)$. Then the associated transform

$$
G(s)=\frac{k}{\alpha^{2}}\left[G_{1}(s)-\frac{1}{2} G_{1}(s-i \alpha)-\frac{1}{2} G_{1}(s+i \alpha)\right]
$$

where $A_{i}$ means the association process for finding associated transform of a function consisting of $i$ variables.

Proof: By equations (1.1) and (1.2), we have

$$
\begin{aligned}
g(t)= & \left.f\left(t_{1}, t_{2}, \ldots, t_{n}\right)\right|_{t_{1}=t_{2}=\ldots=t_{n}=t} \\
= & L_{n}^{-1}\left[F\left(s_{1}, s_{2}, \ldots, s_{n}\right) ; t_{1}, t_{2}, \ldots, t_{n}\right]_{t_{1}=t_{2}=\ldots=t_{n}=t} \\
= & \frac{1}{(2 \pi i)^{n}} \int_{\alpha_{1}-i \infty}^{\alpha_{1}+i \infty} \int_{\alpha_{2}-i \infty}^{\alpha_{2}+i \infty} \ldots \int_{\alpha_{n}-i \infty}^{\alpha_{n}+i \infty} F\left(s_{1}, s_{2}, \ldots, s_{n}\right) \exp \left(\sum_{j=1}^{n} s_{j} t\right) d s_{1} d s_{2} \ldots d s_{n} \\
= & \frac{1}{(2 \pi i)^{n}} \int_{\alpha_{1}-i \infty}^{\alpha_{1}+i \infty} \ldots \int_{\alpha_{n}-i \infty}^{\alpha_{n}+i \infty} \frac{k}{s_{m}\left(s_{m}^{2}+\alpha^{2}\right)} F_{1}\left(s_{1}, \ldots, s_{m-1}, s_{m+1}, \ldots, s_{n}\right) \\
& \quad \exp \left(\sum_{j=1}^{n} s_{j} t\right) d s_{1} d s_{2} \ldots d s_{n}
\end{aligned}
$$




$$
\begin{aligned}
& =\frac{1}{2 \pi i} \int_{\alpha_{m}-i \infty}^{\alpha_{m}+i \infty} \frac{k}{s_{m}\left(s_{m}^{2}+\alpha^{2}\right)} \exp \left(s_{m} t\right) d s_{m} \\
& \frac{1}{(2 \pi i)^{n-1}} \int_{\alpha_{1}-i \infty}^{\alpha_{1}+\infty} \ldots \int_{\alpha_{m-1}-i \infty}^{\alpha_{m-1}+\infty \infty \alpha_{m+1} \alpha_{m+1}+\infty \infty} \ldots \int_{\alpha_{n}}^{\alpha_{n}+\infty \infty} \\
& \cdot F_{1}\left(s_{1}, \ldots, s_{m-1}, s_{m+1}, \ldots, s_{n}\right) \exp \left(\sum_{\substack{j=1 \\
j \neq m}}^{m} s, t\right) \\
& =k L_{1}^{-1}\left[\frac{1}{s_{m}\left(s_{m}^{2}+\alpha^{2}\right)} ; t\right] \\
& \cdot d_{n-1}^{-1}\left[F_{1}\left(s_{1}, \ldots, s_{m-1}, s_{m+1}, \ldots, s_{n}\right) ; t, t, \ldots, t\right] \\
& =k\left[\frac{1-\cos \alpha t}{\alpha^{2}}\right] g_{1}(t) \\
& =\frac{k}{\alpha^{2}}\left[g_{1}(t)-g_{1}(t) \cos \alpha t\right]
\end{aligned}
$$

Taking Laplace Transform of both sides of the equation (2.1) yields

$$
G(s)=\frac{k}{\alpha^{2}}\left[G_{1}(s)-\frac{1}{2} G_{1}(s-i \alpha)-\frac{1}{2} G_{1}\left(s_{i} \alpha\right)\right]
$$

Hence the theorem is proved.

\section{Example 2.1.}

\section{Consider}

$$
F\left(s_{1}, s_{2}, s_{3}\right)=\frac{k}{s_{3}\left(s_{1}+a\right)\left(s_{2}+b\right)\left(s_{3}^{2}+c^{2}\right)}
$$

and let

$$
F_{1}\left(s_{1}, s_{2}\right)=\frac{1}{\left(s_{1}+a\right)\left(s_{2}+b\right)}
$$

Using the table given in [6], we find

$$
F_{1}\left(s_{1}, s_{2}\right) \stackrel{A_{2}}{\longrightarrow} G_{1}(s)=\frac{1}{s+a+b} .
$$

By Theorem 2.1

$$
\begin{aligned}
F\left(s_{1}, s_{2}, s_{3}\right) \stackrel{A_{3}}{\longrightarrow} G(s) & =\frac{k}{c^{2}}\left[\frac{1}{s+a+b}-\frac{1}{2}\left(\frac{1}{s+a+b-i c}\right)-\frac{1}{2}\left(\frac{1}{s+a+b+i c}\right)\right] \\
& =\frac{k}{c^{2}}\left[\frac{1}{s+a+b}-\frac{s+a+b}{(s+a+b)^{2}+c^{2}}\right] \\
& =\frac{k}{(s+a+b)\left[(s+a+b)^{2}+c^{2}\right]} .
\end{aligned}
$$




\section{Example 2.2.}

Let

$$
F\left(s_{1}, s_{2}, s_{3}\right)=\frac{k}{\left[a\left(s_{1}+s_{2}\right)^{2}+b\left(s_{1}+s_{2}\right)+c\right] s_{3}\left(s_{3}^{2}+d^{2}\right)}
$$

and take

$$
F_{1}\left(s_{1}, s_{2}\right)=\frac{1}{a\left(s_{1}+s_{2}\right)^{2}+b\left(s_{1}+s_{2}\right)+c}
$$

Use of the table in [6] gives

$$
F_{1}\left(s_{1}, s_{2}\right) \stackrel{A_{2}}{\longrightarrow} G_{1}(s)=\frac{1}{a s^{2}+b s+c}
$$

Thus Theorem 2.1 yields

$$
\begin{aligned}
F\left(s_{1}, s_{2}, s_{3}\right) \stackrel{A_{3}}{\longrightarrow} G(s)=\frac{k}{2 d^{2}} & {\left[\frac{2}{a s^{2}+b s+c}-\frac{1}{a(s-i d)^{2}+b(s-i d)+c}\right.} \\
& \left.-\frac{1}{a(s+i d)^{2}+b(s+i d)+c}\right] .
\end{aligned}
$$

Theorem 2.2. If a given function $F\left(s_{1}, s_{2}, \ldots, s_{n}\right)$ can be factored in the form

$$
F\left(s_{1}, s_{2}, \ldots, s_{n}\right)=\frac{k}{s_{m}\left(s_{m}+a\right)\left(s_{m}+b\right)} F_{1}\left(s_{1}, \ldots, s_{m-1}, s_{m+1}, \ldots, s_{n}\right)
$$

and if

$$
F_{1}\left(s_{1}, \ldots, s_{m-1}, s_{m+1}, \ldots, s_{n}\right) \stackrel{A_{n-1}}{\longrightarrow} G_{1}(s)
$$

then the associated transform

$$
G(s)=\frac{k}{a b} G_{1}(s)+\frac{k}{a b(a-b)}\left[b G_{1}(s+a)-a G_{1}(s+b)\right] .
$$

Proof: By equations (1.1) and (1.2), we get

$$
\begin{aligned}
g(t) & =\left.f\left(t_{1}, t_{2}, \ldots, t_{n}\right)\right|_{t_{1}=t_{2}=\cdots=t_{n}=t} \\
& =\left.L_{n}^{-1}\left[F\left(s_{1}, s_{2}, \ldots, s_{n}\right) ; t_{1}, t_{2}, \ldots, t_{n}\right]\right|_{t_{1}=t_{2}=\cdots=t_{n}=t}
\end{aligned}
$$




$$
\begin{aligned}
& =\frac{1}{(2 \pi i)^{n}} \int_{\alpha_{1}-i \infty}^{\alpha_{1}+i \infty} \cdots \int_{\alpha_{n}-i \infty}^{\alpha_{n}+i \infty} F\left(s_{1}, s_{2}, \ldots, s_{n}\right) \\
& \cdot \exp \left(\sum_{j=1}^{n} s_{j} t\right) d s_{1} \ldots d s_{n} \\
& =\frac{1}{(2 \pi i)^{n}} \int_{\alpha_{1}-i \infty}^{\alpha_{1}+i \infty} \ldots \int_{\alpha_{n}-i \infty}^{\alpha_{n}+i \infty} \frac{k}{s_{m}\left(s_{m}+a\right)\left(s_{m}+b\right)} F_{1}\left(s_{1}, \ldots, s_{m-1}, s_{m+1}, \ldots, s_{n}\right) \\
& \cdot \exp \left(\sum_{j=1}^{n} s_{j} t\right) d s_{1} \ldots d s_{n} \\
& =\frac{1}{2 \pi i} \int_{\alpha_{m}-i \infty}^{\alpha_{m}+i \infty} \frac{k}{s_{m}\left(s_{m}+a\right)\left(s_{m}+b\right)} \exp \left(s_{m} t\right) d s_{m} \\
& \frac{1}{(2 \pi i)^{n-1}} \int_{\alpha_{1}-i \infty}^{\alpha_{1}+i \infty} \cdots \int_{\alpha_{m-1}-i \infty}^{\alpha_{m-1}+i \infty} \int_{\alpha_{m+1}-i \infty}^{\alpha_{m+1}+i \infty} \cdots \int_{\alpha_{n}-i \infty}^{\alpha_{n}+i \infty} \\
& \cdot F_{1}\left(s_{1}, \ldots, s_{m-1}, s_{m+1}, \ldots, s_{n}\right) \exp \left(\sum_{\substack{j=1 \\
j \neq m}}^{n} s_{j} t\right) \\
& \cdot d s_{1} \ldots d s_{m-1} d s_{m+1} \ldots d s_{n} \\
& =k L_{1}^{-1}\left[\frac{1}{s_{m}\left(s_{m}+a\right)\left(s_{m}+b\right)} ; t\right] \\
& \text { - } L_{n-1}^{-1}\left[F_{1}\left(s_{1}, \ldots, s_{m-1}, s_{m+1}, \ldots, s_{n}\right) ; t, t, \ldots, t\right] \\
& =k\left[\frac{1}{a b}+\frac{1}{a b(a-b)}\left(b e^{-a t}-a e^{-b t}\right)\right] g_{1}(t) \\
& =\frac{k}{a b} g_{1}(t)+\frac{k}{a b(a-b)}\left[b e^{-a t} g_{1}(t)-a e^{-b t} g_{1}(t)\right]
\end{aligned}
$$

On taking Laplace transform of both sides of equation (2.2), we obtain

$$
G(s)=\frac{k}{a b} G_{1}(s)+\frac{k}{a b(a-b)}\left[b G_{1}(s+a)-a G_{1}\left(s_{b}\right)\right]
$$

This establishes the theorem.

\section{Example 2.3.}

\section{Suppose}

$$
F\left(s_{1}, s_{2}, s_{3}\right)=\frac{k}{\left(s_{1}+s_{2}+a\right) s_{3}\left(s_{3}+b\right)\left(s_{3}+c\right)}
$$


and let

$$
F_{1}\left(s_{1}, s_{2}\right)=\frac{1}{s_{1}+s_{2}+a} .
$$

From the table shown in [5]

$$
F_{1}\left(s_{1}, s_{2}\right) \stackrel{A_{2}}{\longrightarrow} G_{1}(s)=\frac{1}{s+a} .
$$

Then by using Theorem 2.2, we get

$$
\begin{aligned}
F\left(s_{1}, s_{2}, s_{3}\right) \stackrel{A_{3}}{\longrightarrow} G(s) & =\frac{k}{b c(s+a)}+\frac{k}{b c(b-c)}\left[\frac{c}{s+a+b}-\frac{b}{s+a+c}\right] \\
& =\frac{k}{b c(s+a)}-\frac{k}{b c(c-b)}\left[\frac{(c-b)(s+a+b+c)}{(s+a+b)(s+a+c)}\right] \\
& =\frac{k}{b c(s+A)}-\frac{k(s+a+b+c)}{b c(s+a+b)(s+a+c)}
\end{aligned}
$$

\section{Example 2.4.}

Consider

and take

$$
F\left(s_{1}, s_{2}, s_{3}\right)=\frac{k}{\left(s_{1}+a\right)\left(s_{2}+b\right) s_{3}\left(s_{3}+c\right)\left(s_{3}+d\right)}
$$

$$
F_{1}\left(s_{1}, s_{2}\right)=\frac{1}{\left(s_{1}+a\right)\left(s_{2}+b\right)}
$$

From the table shown in [6]

$$
F_{1}\left(s_{1}, s_{2}\right) \stackrel{A_{2}}{\longrightarrow} G_{1}(s)=\frac{1}{s+a+b}
$$

Then by using Theorem 2.2 , we get

$$
\begin{aligned}
F\left(s_{1}, s_{2}, s_{3}\right) \stackrel{A_{3}}{\longrightarrow} G(s) & =\frac{k}{c d} \frac{1}{s+a+b}+\frac{k}{c d(c-d)}\left[\frac{d}{s+a+b+c}-\frac{c}{s+a+b+d}\right] \\
& =\frac{k}{c d}\left[\frac{1}{s+a+b}-\frac{s+a+b+c+d}{(s+a+b+c)(s+a+b+d)}\right]
\end{aligned}
$$

Theorem 2.3. If a function $F\left(s_{1}, s_{2}, \ldots, s_{n}\right)$ is of the form

$$
F\left(s_{1}, s_{2}, \ldots, s_{n}\right)=\frac{k\left(s_{m}^{2}+a s_{m}+b\right)}{s_{m}\left(s_{m}^{2}-\alpha^{2}\right)} F_{1}\left(s_{1}, \ldots, s_{m-1}, s_{m+1}, \ldots, s_{n}\right)
$$

with $F_{1}\left(s_{1}, \ldots, s_{m-1}, s_{m+1}, \ldots, s_{n}\right) \stackrel{A_{n-1}}{\longrightarrow} G_{1}(s)$. Then

$$
F\left(s_{1}, s_{2}, \ldots, s_{n}\right) \stackrel{A_{n}}{\longrightarrow} G(s)
$$


where $G(s)=\frac{k}{2 \alpha^{2}}\left[\left(\alpha^{2}+a \alpha+b\right) G_{1}(s-a)+\left(\alpha^{2}-a \alpha+b\right) G_{1}(s+a)-2 b G_{1}(s)\right]$.

Proof: By definitions (1.1) and (1.2),

$$
\begin{aligned}
& g(t)=\left.f\left(t_{1}, t_{2}, \ldots, t_{n}\right)\right|_{t_{1}=t_{2}=\cdots=t_{n}=t} \\
& =L_{n}^{-1}\left[F\left(s_{1}, s_{2}, \ldots, s_{n}\right) ; t, t, \ldots, t\right] \\
& =\frac{1}{(2 \pi i)^{n}} \int_{\alpha_{1}-i \infty}^{\alpha_{1}+i \infty} \ldots \int_{\alpha_{n}-i \infty}^{\alpha_{n}+i \infty} \frac{k\left(s_{m}^{2}+a s_{m}+b\right)}{s_{m}\left(s_{m}^{2}-\alpha^{2}\right)} F_{1}\left(s_{1}, \ldots, s_{m-1}, s_{m+1}, \ldots, s_{n}\right) \\
& \cdot \exp \left(\sum_{j=1}^{n} s_{j} t\right) d s_{1} d s_{2} \ldots d s_{n} \\
& =\frac{1}{2 \pi i} \int_{\alpha_{m}-i \infty}^{\alpha_{m}+i \infty} \frac{k\left(s_{m}^{2}+a s_{m}+b\right)}{s_{m}\left(s_{m}^{2}-\alpha^{2}\right)} \exp \left(s_{m} t\right) d s_{m} \\
& \frac{1}{(2 \pi i)^{n-1}} \int_{\alpha_{1}-i \infty}^{\alpha_{1}+i \infty} \cdots \int_{\alpha_{m-1}-i \infty}^{\alpha_{m-1}+i \infty} \int_{\alpha_{m+1}-i \infty}^{\alpha_{m+1}+i \infty} \cdots \int_{\alpha_{n}-i \infty}^{\alpha_{n}+i \infty} \\
& \cdot F_{1}\left(s_{1}, \ldots, s_{m-1}, s_{m+1}, \ldots, s_{n}\right) \exp \left(\sum_{\substack{j=1 \\
j \neq m}}^{n} s_{j} t\right) \\
& d s_{1} \ldots d s_{m-1} d s_{m+1} \ldots d s_{n} \\
& =k L_{1}^{-1}\left[\frac{s_{m}^{2}+a s_{m}+b}{s_{m}\left(s_{m}^{2}-\alpha^{2}\right)} ; t\right] L_{n-1}^{-1}\left[F_{1}\left(s_{1}, \ldots, s_{m-1}, s_{m+1}, \ldots, s_{n}\right) ; t, t, \ldots, t\right] \\
& =k\left[\frac{a}{\alpha} \sinh \alpha t+\frac{\alpha^{2}+b}{\alpha^{2}} \cosh \alpha t-\frac{b}{\alpha^{2}}\right] g_{1}(t) \\
& =\frac{k a}{\alpha} g_{1}(t) \sinh \alpha t+\frac{k\left(\alpha^{2}+b\right)}{\alpha^{2}} g_{1}(t) \cosh \alpha t-\frac{k b}{\alpha^{2}} g_{1}(t)
\end{aligned}
$$

Taking Laplace transform on both sides of (2.3)

$$
\begin{aligned}
G(s) & =\frac{k a}{2 \alpha}\left[G_{1}(s-a)-G_{1}(s+a)\right]+\frac{k\left(\alpha^{2}+b\right)}{2 \alpha^{2}}\left[G_{1}(s-a)+G_{1}(s+a)\right]-\frac{b}{\alpha^{2}} G_{1}(s) \\
& =\frac{k}{2 \alpha^{2}}\left[\left(\alpha^{2}+a \alpha+b\right) G_{1}(s-a)+\left(\alpha^{2}-a \alpha+b\right) G_{1}(s+a)-2 b G_{1}(s)\right] .
\end{aligned}
$$

\section{Example 2.5.}

Suppose

$$
F\left(s_{1}, s_{2}, s_{3}\right)=\frac{k\left(s_{3}^{2}+a s_{3}+b\right)}{\left(s_{1}+\alpha\right)\left(s_{2}+\alpha\right)\left(s_{1}+s_{2}+\beta\right) s_{3}\left(s_{3}^{2}-\gamma^{2}\right)}
$$

and say

$$
F_{1}\left(s_{1}, s_{2}\right)=\frac{1}{\left(s_{1}+\alpha\right)\left(s_{2}+\alpha\right)\left(s_{1}+s_{2}+\beta\right)}
$$


Use of the table given in [6]

$$
F_{1}\left(s_{1}, s_{2}\right) \stackrel{A_{2}}{\longrightarrow} G_{1}(s)=\frac{1}{(s+2 \alpha)(s+\beta)} .
$$

Application of Theorem 2.3 gives

$$
G(s)=\frac{k}{2 \gamma^{2}}\left[\frac{\gamma^{2}+a \gamma+b}{(s-a+2 \alpha)(s-a+\beta)}+\frac{\gamma^{2}-a \gamma+b}{(s+a+2 \alpha)(s+a+\beta)}-\frac{2 b}{(s+2 \alpha)(s+\beta)}\right] .
$$

\section{Example 2.6.}

Consider

$$
F\left(s_{1}, s_{2}, s_{3}\right)=\frac{k\left(s_{3}^{2}+a s_{3}+b\right)}{s_{1} s_{2} s_{3}\left\{\left(s_{1}+s_{2}\right)^{2}+c\left(s_{1}+s_{2}\right)+d\right\}\left(s_{3}^{2}-\alpha^{2}\right)}
$$

and let

$$
F_{1}\left(s_{1}, s_{2}\right)=\frac{1}{s_{1} s_{2}\left\{\left(s_{1}+s_{2}\right)^{2}+c\left(s_{1}+s_{2}\right)+d\right\}} .
$$

Then by using the result shown in [6],

$$
F_{1}\left(s_{1}, s_{2}\right) \stackrel{A_{2}}{\longrightarrow} G_{1}(s)=\frac{1}{s\left(s^{2}+c s+d\right)} .
$$

Theorem 2.3 gives

$$
\begin{aligned}
G(s)=\frac{k}{2 \alpha^{2}}[ & \frac{\alpha^{2}+a \alpha+b}{(s-a)\left\{(s-a)^{2}+c(s-a)+d\right\}}+\frac{\alpha^{2}-a \alpha+b}{(s+a)\left\{(s+a)^{2}+c(s+a)+d\right\}} \\
& \left.-\frac{2 b}{s\left(s^{2}+c s+d\right)}\right] .
\end{aligned}
$$

Theorem 2.4. If $F\left(s_{1}, s_{2}, \ldots, s_{n}\right)$ can be expressed in the following form

$$
F\left(s_{1}, s_{2}, \ldots, s_{n}\right)=\frac{k\left(s_{m}+a\right)}{s_{m}^{2}\left(s_{m}^{2}+\alpha^{2}\right)} F_{1}\left(s_{1}, \ldots, s_{m-1}, s_{m+1}, \ldots, s_{n}\right)
$$

where

$$
F_{1}\left(s_{1}, \ldots, s_{m-1}, s_{m+1}, \ldots, s_{n}\right) \stackrel{A_{n-1}}{\longrightarrow} G_{1}(s)
$$

Then the associated transform

$$
\begin{aligned}
G(s) & =k\left[\frac{1}{\alpha^{2}} G_{1}(s)-\frac{a}{\alpha^{2}} \frac{d}{d s} G_{1}(d)-\frac{1}{2 \alpha^{2}}\left(1+\frac{a}{i \alpha}\right) G_{1}(s-i \alpha)\right. \\
& \left.-\frac{1}{2 \alpha^{2}}\left(1-\frac{a}{i \alpha}\right) G_{1}(s+i \alpha)\right]
\end{aligned}
$$

Proof: By definitions (1.1) and (1.2),

$$
\begin{aligned}
g(t) & =\left.L_{n}^{-1}\left[F\left(s_{1}, s_{2}, \ldots, s_{n}\right) ; t_{1}, t_{2}, \ldots, t_{n}\right]\right|_{t_{1}=t_{2}=\cdots=t_{n}=t} \\
& =L_{1}^{-1}\left[\frac{k\left(s_{m}+a\right)}{s_{m}^{2}\left(s_{m}^{2}+\alpha^{2}\right)} ; t\right] L_{n-1}^{-1}\left[F_{1}\left(s_{1}, \ldots, s_{m-1}, s_{m+1}, \ldots, s_{n}\right) ; t, t, \ldots, t\right] .
\end{aligned}
$$

By the results of inverse Laplace transform shown in [8], we obtain 


$$
g(t)=k\left[\frac{1}{\alpha^{2}}+\frac{a t}{\alpha^{2}}-\frac{1}{\alpha^{2}} \cos \alpha t-\frac{a}{\alpha^{3}} \sin \alpha t\right] g_{1}(t)
$$

On taking Laplace transform of both sides of (2.4)

$$
\begin{aligned}
L[g(t) ; s]=L & {\left[\frac{k}{\alpha^{2}} g_{1}(t)+\frac{k a t}{\alpha^{2}} g_{1}(t)-\frac{k}{\alpha^{2}} g_{1}(t) \cos \alpha t\right.} \\
& \left.-\frac{k a}{\alpha^{3}} g_{1}(t) \sin \alpha t\right] .
\end{aligned}
$$

We establish the theorem. That is,

Simplifying,

$$
\begin{aligned}
G(s)= & \frac{k}{\alpha^{2}} G_{1}(s)-\frac{k a}{\alpha^{2}} \frac{d}{d s} G_{1}(s)-\frac{k}{2 \alpha^{2}}\left[G_{1}(s-i \alpha)+G_{1}(s+i \alpha)\right] \\
& \frac{k a}{2 i \alpha^{3}}\left[G_{1}(s-i \alpha)-G_{1}(s+i \alpha)\right] .
\end{aligned}
$$

$$
\begin{aligned}
G(s)=k & {\left[\frac{1}{\alpha^{2}} G_{1}(s)-\frac{a}{\alpha^{2}} \frac{d}{d s} G_{1}(s)-\frac{1}{2 \alpha^{2}}\left(1+\frac{a}{i \alpha}\right) G_{1}(s-i \alpha)\right.} \\
& \left.-\frac{1}{2 \alpha^{2}}\left(1-\frac{a}{i \alpha}\right) G_{1}(s+i \alpha)\right]
\end{aligned}
$$

\section{Example 2.7.}

\section{Consider}

$$
F\left(s_{1}, s_{2}, s_{3}\right)=\frac{k\left(s_{3}+a\right)}{s_{3}^{2}\left(s_{3}^{2}+\alpha^{2}\right)\left(s_{1}+s_{2}+b\right)} .
$$

Then we find

$$
F_{1}\left(s_{1}, s_{2}\right)=\frac{1}{s_{1}+s_{2}+b} \stackrel{A_{2}}{\longrightarrow} G_{1}(s)=\frac{1}{s+b} .
$$

Use of Theorem 2.4 gives

$$
\begin{aligned}
G(s)=k & {\left[\frac{1}{\alpha^{2}(s+b)}+\frac{a}{\alpha^{2}(s+b)^{2}}-\left(1+\frac{a}{i \alpha}\right) \frac{1}{2 \alpha^{2}(s-i \alpha+b)}\right.} \\
& \left.-\left(1-\frac{a}{i \alpha}\right) \frac{1}{2 \alpha^{2}(s+i \alpha+b)}\right] \\
= & \frac{k}{2 \alpha^{2}}\left[\frac{2}{s+b}+\frac{2 a}{(s+b)^{2}}-\frac{\alpha-a i}{\alpha(s-i \alpha+b)}-\frac{\alpha+a i}{\alpha(s+i \alpha+b)}\right] \\
= & \frac{k}{2 \alpha^{2}}\left[\frac{2(s+a+b)}{(s+b)^{2}}-\frac{2 \alpha(s+a+b)}{\alpha(s+b+i \alpha)(s+b-i \alpha)}\right] \\
= & \frac{k(s+a+b)}{\alpha^{2}}\left[\frac{1}{(s+b)^{2}}-\frac{1}{(s+b)^{2}+\alpha^{2}}\right]
\end{aligned}
$$

\section{Example 2.8.}

Suppose

$$
F\left(s_{1}, s_{2}, s_{3}\right)=\frac{k\left(s_{3}+a\right)}{s_{1} s_{2} s_{3}^{2}\left(s_{1}+s_{2}+b\right)\left(s_{3}^{2}+\alpha^{2}\right)} .
$$


Then we can find

$$
F_{1}\left(s_{1}, s_{2}\right)=\frac{1}{s_{1} s_{2}\left(s_{1}+s_{2}+b\right)} \stackrel{A_{2}}{\longrightarrow} G_{1}(s)=\frac{1}{s(s+b)} .
$$

Using Theorem 2.4, we get

$$
\begin{aligned}
G(s)=\frac{k}{2 \alpha^{2}} & {\left[\frac{2}{s(s+b)}+\frac{2 a(2 s+b)}{s^{2}(s+b)^{2}}-\frac{\alpha-a i}{\alpha(s-i \alpha)(s-i \alpha+b)}\right.} \\
& \left.-\frac{\alpha+a i}{\alpha(s+i \alpha)(s+i \alpha+b)}\right] .
\end{aligned}
$$

Theorem 2.5. If a function $F\left(s_{1}, s_{2}, \ldots, s_{n}\right)$ can be expressed in the form

$$
F\left(s_{1}, s_{2}, \ldots, s_{n}\right)=\frac{\left(s_{m}^{3}+a s_{m}^{2}+b s_{m}+c\right) k}{\left(s_{m}+\alpha\right)\left(s_{m}+\beta\right)\left(s_{m}+\gamma\right)\left(s_{m}+\delta\right)} F\left(s_{1}, \ldots, s_{m-1}, s_{m+1}, \ldots, s_{n}\right),
$$

then its associated transform

$$
\begin{aligned}
G(s)=-k & {\left[\frac{\alpha^{3}-a \alpha^{2}+b \alpha-c}{(\beta-\alpha)(\gamma-\alpha)(\delta-\alpha)} G_{1}(s+\alpha)+\frac{\beta^{3}-a \beta^{2}+b \beta-c}{(\alpha-\beta)(\gamma-\beta)(\delta-\beta)} G_{1}(s+\beta)\right.} \\
+ & \left.\frac{\gamma^{3}-a \gamma^{2}+b \gamma-c}{(\alpha-\gamma)(\beta-\gamma)(\delta-\gamma)} G_{1}(s+\gamma)+\frac{\delta^{3}-a \delta^{2}+b \delta-c}{(\alpha-\delta)(\beta-\delta)(\gamma-\delta)} G_{1}(s+\delta)\right]
\end{aligned}
$$

where $G_{1}(s)$ is the associated transform of $F_{1}$.

Proof: By definitions (1.1) and (1.2)

$$
\begin{aligned}
g(t)= & L_{n}^{-1}\left[F\left(s_{1}, s_{2}, \ldots, s_{n}\right) ; t, t, \ldots, t\right] \\
= & k L_{1}^{-1}\left[\frac{s_{m}^{3}+a s_{m}^{2}+b s_{m}+c}{\left(s_{m}+\alpha\right)\left(s_{m}+\beta\right)\left(s_{m}+\gamma\right)\left(s_{m}+\delta\right)} ; t\right] \\
& \cdot L_{n-1}^{-1}\left[F_{1}\left(s_{1}, \ldots, s_{m-1}, s_{m+1}, \ldots, s_{n}\right) ; t, t, \ldots, t\right]
\end{aligned}
$$

Referring to the results given in [8],

$$
\begin{aligned}
g(t)=-k & {\left[\frac{\alpha^{3}-a \alpha^{2}+b \alpha-c}{(\beta-\alpha)(\gamma-\alpha)(\delta-\alpha)} e^{-\alpha t}+\frac{\beta^{3}-a \beta^{2}+b \beta-c}{(\alpha-\beta)(\gamma-\beta)(\delta-\beta)} e^{-\beta t}\right.} \\
& \left.+\frac{\gamma^{3}-a \gamma^{2}+b \gamma-c}{(\alpha-\gamma)(\beta-\gamma)(\delta-\gamma)} e^{-\gamma t}+\frac{\delta^{3}-a \delta^{2}+b \delta-c}{(\alpha-\delta)(\beta-\delta)(\gamma-\delta)} e^{-\delta t}\right] g_{1}(t) .
\end{aligned}
$$

Taking Laplace transform on both sides of equation (2.5),

$$
\begin{aligned}
L[g(t) ; s]=-k L\left[\frac{\alpha^{3}-a \alpha^{2}+b \alpha-c}{(\beta-\alpha)(\gamma-\alpha)(\delta-\alpha)} e^{-\alpha t} g_{1}(t)+\frac{\beta^{3}-a \beta^{2}+b \beta-c}{(\alpha-\beta)(\gamma-\beta)(\delta-\beta)} e^{-\beta t} g_{1}(t)\right. \\
\left.+\frac{\gamma^{3}-a \gamma^{2}+b \gamma-c}{(\alpha-\gamma)(\beta-\gamma)(\delta-\gamma)} e^{-\gamma t} g_{1}(t)+\frac{\delta^{3}-a \delta^{2}+b \delta-c}{(\alpha-\delta)(\beta-\delta)(\gamma-\delta)} e^{-\delta t} g_{1}(t) ; s\right]
\end{aligned}
$$

We finally obtain

$$
\begin{aligned}
G(s)=-k & {\left[\frac{\alpha^{3}-a \alpha^{2}+b \alpha-c}{(\beta-\alpha)(\gamma-\alpha)(\delta-\alpha)} G_{1}(s+\alpha)+\frac{\beta^{3}-a \beta^{2}+b \beta-c}{(\alpha-\beta)(\gamma-\beta)(\delta-\beta)} G_{1}(s+\beta)\right.} \\
+ & \left.\frac{\gamma^{3}-a \gamma^{2}+b \gamma-c}{(\alpha-\gamma)(\beta-\gamma)(\delta-\gamma)} G_{1}(s+\gamma)+\frac{\delta^{3}-a \delta^{2}+b \delta-c}{(\alpha-\delta)(\beta-\delta)(\gamma-\delta)} G_{1}(s+\delta)\right]
\end{aligned}
$$




\section{Example 2.9.}

Consider

$$
F\left(s_{1}, s_{2}, s_{3}\right)=\frac{k\left(s_{3}^{3}+a s_{3}^{2}+b s_{3}+c\right)}{\left(s_{1}+d\right)\left(s_{2}+e\right)\left(s_{3}+\alpha\right)\left(s_{3}+\beta\right)\left(s_{3}+\gamma\right)\left(s_{3}+\delta\right)} .
$$

Direct use of the table given in [7], we find

$$
F_{1}\left(s_{1}, s_{2}\right)=\frac{1}{\left(s_{1}+d\right)\left(s_{2}+e\right)} \stackrel{A_{2}}{\longrightarrow} G_{1}(s)=\frac{1}{s+d+e} .
$$

Thus, by Theorem 2.5

$$
\begin{aligned}
F\left(s_{1}, s_{2}, s_{3}\right) \stackrel{A_{3}}{\longrightarrow} G(s)=-k\left[\frac{\alpha^{3}-a \alpha^{2}+b \alpha-c}{(\beta-\alpha)(\gamma-\alpha)(\delta-\alpha)(s+\alpha+d+e)}\right. \\
+\frac{\beta^{3}-a \beta^{2}+b \beta-c}{(\alpha-\beta)(\gamma-\beta)(\delta-\beta)(s+\beta+d+e)} \\
+\frac{\gamma^{3}-a \gamma^{2}+b \gamma-c}{(\alpha-\gamma)(\beta-\gamma)(\delta-\gamma)(s+\gamma+d+e)} \\
\left.+\frac{\delta^{3}-a \delta^{2}+b \delta-c}{(\alpha-\delta)(\beta-\delta)(\gamma-\delta)(s+\delta+d+e)}\right] .
\end{aligned}
$$

Example 2.10.

\section{Suppose}

$$
F\left(s_{1}, s_{2}, s_{3}\right)=\frac{k\left(s_{3}^{3}+a s_{3}^{2}+b s_{3}+c\right)}{\left(s_{1}+s_{2}+d\right)\left(s_{3}+\alpha\right)\left(s_{3}+\beta\right)\left(s_{3}+\gamma\right)\left(s_{3}+\delta\right)} .
$$

Then we find

$$
F_{1}\left(s_{1}, s_{2}\right)=\frac{1}{s_{1}+s_{2}+d} \stackrel{A_{2}}{\longrightarrow} G_{1}(s)=\frac{1}{s+d} .
$$

Use of Theorem 2.5 gives

$$
\begin{aligned}
F\left(s_{1}, s_{2}, s_{3}\right) \stackrel{A_{s}}{\longrightarrow} G(s)=- & k\left[\frac{a^{3}-a \alpha^{2}+b \alpha-c}{(\beta-\alpha)(\gamma-\alpha)(\delta-\alpha)(s+\alpha+d)}\right. \\
& +\frac{\beta^{2}-a \beta^{2}+b \beta-c}{(\alpha-\beta)(\gamma-\beta)(\delta-\beta)(s+\beta+d)} \\
& +\frac{\gamma^{3}-a \gamma^{2}+b \gamma-c}{(\alpha-\gamma)(\beta-\gamma)(\delta-\gamma)(s+\gamma+d)} \\
& \left.+\frac{\delta^{3}-a \delta^{2}+b \delta-c}{(\alpha-\delta)(\beta-\delta)(\gamma-\delta)(s+\delta+d)}\right]
\end{aligned}
$$

Theorem 2.6. If a function

$$
F\left(s_{1}, s_{2}, \ldots, s_{n}\right)=\frac{k}{\left(s_{m}+\alpha\right)^{2}\left(s_{m}+\beta\right)} F\left(s_{1}, \ldots, s_{m-1}, s_{m+1}, \ldots, s_{n}\right),
$$

then

$$
G(s)=\frac{k}{(\beta-\alpha)^{2}}\left[G_{1}(s+\beta)-(\beta-\alpha) \frac{d}{d s} G_{1}(s+\alpha)-G_{1}(s+\alpha)\right] .
$$

Proof: By definitions (1.1) and (1.2)

$$
g(t)=L_{n}^{-1}\left[F\left(s_{1}, s_{2}, \ldots, s_{n}\right) ; t, t, \ldots, t\right]
$$




$$
\begin{aligned}
= & k L_{1}^{-1}\left[\frac{1}{\left(s_{m}+\alpha\right)^{2}\left(s_{m}+\beta\right)} ; t\right] \\
& \cdot L_{n-1}^{-1}\left[F_{1}\left(s_{1}, \ldots, s_{m-1}, s_{m+1}, \ldots, s_{n}\right) ; t, t, \ldots, t\right] \\
= & \frac{k}{(\beta-\alpha)^{2}}\left[e^{-\beta t}+(\beta-\alpha) t e^{-\alpha t}-e^{-\alpha t}\right] g_{1}(t) .
\end{aligned}
$$

On taking Laplace transform on both sides, one obtains,

\section{Example 2.11.}

$$
G(s)=\frac{k}{(\beta-\alpha)^{2}}\left[G_{1}(s+\beta)-(\beta-\alpha) \frac{d}{d s} G_{1}(s+\alpha)-G_{1}(s+\alpha)\right]
$$

Let

$$
F\left(s_{1}, s_{2}, s_{3}\right)=\frac{k}{\left(s_{1}+a\right)\left(s_{2}+b\right)\left(s_{3}+c\right)\left(s_{3}+d\right)^{2}}
$$

Then

$$
F_{1}\left(s_{1}, s_{2}\right)=\frac{1}{\left(s_{1}+a\right)\left(s_{2}+b\right)} \stackrel{A_{2}}{\longrightarrow} G_{1}(s)=\frac{1}{s+a+b} .
$$

Thus, the application of Theorem 2.6 shows

\section{Example 2.12.}

$$
\begin{aligned}
F\left(s_{1}, s_{2}, s_{3}\right) \stackrel{A_{3}}{\longrightarrow} G(s)= & \frac{k}{(c-d)^{2}}\left[\frac{1}{s+a+b+c}\right. \\
& \left.+\frac{(c-d)}{(s+a+b+d)^{2}}-\frac{1}{s+a+b+d}\right] .
\end{aligned}
$$

Suppose

$$
F\left(s_{1}, s_{2}, s_{3}\right)=\frac{k}{\left\{a\left(s_{1}+s_{2}\right)^{2}+b\left(s_{1}+s_{2}\right)+c\right\}\left(s_{3}+\alpha\right)^{2}\left(s_{3}+\beta\right)} .
$$

Thus

$$
F_{1}\left(s_{1}, s_{2}\right)=\frac{1}{\left\{a\left(s_{1}+s_{2}\right)^{2}+b\left(s_{1}+s_{2}\right)+c\right\}} \stackrel{A_{2}}{\longrightarrow} G_{1}(s)=\frac{1}{a s^{2}+b s+c}
$$

and using Theorem 2.6, we get

$$
\begin{aligned}
F\left(s_{1}, s_{2}, s_{3}\right) \stackrel{A_{3}}{\longrightarrow} G(s)= & \frac{k}{(\beta-\alpha)^{2}}\left[\frac{1}{a(s+\beta)^{2}+b(s+\beta)+c}\right. \\
& \left.\quad+\frac{(\beta-\alpha)(2 a s+2 a \beta+b)}{\left\{a(s+\beta)^{2}+b(s+\beta)+c\right\}^{2}}-\frac{1}{a(s+\alpha)^{2}+b(s+\alpha)+c}\right] .
\end{aligned}
$$

Following analogous arguments, it is easy to prove the following results.

\section{Theorem 2.7. If}

$$
F\left(s_{1}, s_{2}, \ldots, s_{n}\right)=\frac{\left(s_{m}^{2}-2 \alpha^{2}\right) k}{s_{m}\left(s_{m}-4 \alpha^{2}\right)} F_{1}\left(s_{1}, \ldots, s_{m-1}, s_{m+1}, \ldots, s_{n}\right)
$$

then its associated transform

$$
G(s)=\frac{k}{4}\left[G_{1}(s-2 \alpha)+2 G_{1}(s)+G_{1}(s+2 \alpha)\right] .
$$




\section{Example 2.13.}

Consider

$$
F\left(s_{1}, s_{2}, s_{3}\right)=\frac{k\left(s_{3}^{2}-2 \alpha^{2}\right)}{\left(s_{1}+a\right)\left(s_{2}+b\right) s_{3}\left(s_{3}-4 \alpha^{2}\right)}
$$

Then

\section{Example 2.14.}

$$
\begin{aligned}
G(s) & =\frac{k}{4}\left[\frac{1}{s+a+b-2 \alpha}+\frac{2}{s+a+b}+\frac{1}{s+a+b+2 \alpha}\right] \\
& =\frac{k\left\{(s+a+b)^{2}-2 \alpha^{2}\right\}}{\left\{(s+a+b)^{2}-4 \alpha^{2}\right\}(s+a+b)} .
\end{aligned}
$$

Considering

$$
F\left(s_{1}, s_{2}, s_{3}\right)=\frac{\left(s_{3}^{2}-2 \alpha^{2}\right) k}{\left\{a\left(s_{1}+s_{2}\right)^{2}+b\left(s_{1}+s_{2}\right)+c\right\} s_{3}\left(s_{3}-4 \alpha^{2}\right)}
$$

We obtain

or,

$$
\begin{aligned}
G(s)=\frac{k}{4} & {\left[\frac{1}{a(s-2 \alpha)^{2}+b(s-2 \alpha)+c}+\frac{2}{a s^{2}+b s+c}\right.} \\
& \left.+\frac{1}{a(s+2 \alpha)^{2}+b(s+2 \alpha)+c}\right]
\end{aligned}
$$

$$
G(s)=\frac{k}{2}\left[\frac{(a+b) s+4 \alpha^{2}+c}{\left\{a(s-2 \alpha)^{2}+b(s-2 \alpha)+c\right\}\left\{a(s+2 \alpha)^{2}+b(s+2 \alpha)+c\right\}}\right.
$$

\section{Theorem 2.8. If}

$$
\left.+\frac{1}{a s^{2}+b s+c}\right] \text {. }
$$

$$
F\left(s_{1}, s_{2}, \ldots, s_{n}\right)=\frac{k}{\left(s_{m}+\alpha\right)\left(s_{m}^{2}-\beta^{2}\right)} F_{1}\left(s_{1}, \ldots, s_{m-1}, s_{m+1}, \ldots, s_{n}\right)
$$

then its associated transform

$$
G(s)=\frac{k}{2\left(\beta^{2}-\alpha^{2}\right)}\left[-2 G_{1}(s+\alpha)+\left(1-\frac{\alpha}{\beta}\right) G_{1}(s-\beta)+\left(1+\frac{\alpha}{\beta}\right) G_{1}(s+\beta)\right] .
$$

\section{Example 2.15.}

Let

$$
F\left(s_{1}, s_{2}, s_{3}\right)=\frac{k}{\left(s_{1}+a\right)\left(s_{2}+b\right)\left(s_{3}+\alpha\right)\left(s_{3}^{2}-\beta^{2}\right)} .
$$

Then, direct application of Theorem 2.8 gives,

$$
\begin{aligned}
G(s) & =\frac{1}{2\left(\beta^{2}-\alpha^{2}\right)}\left[-\frac{2}{(s+a+b+\alpha)}+\frac{(\beta-\alpha)}{\beta(s+a+b-\beta)}+\frac{(\beta+\alpha)}{\beta(s+a+b+\beta)}\right] \\
& =\frac{k}{\beta^{2}-\alpha^{2}}\left[\frac{s+a+b-\alpha}{(s+a+b)^{2}-\beta^{2}}-\frac{1}{s+a+b+\alpha}\right] \\
& =\frac{k}{\left\{(s+a+b)^{2}-\beta^{2}\right\}(s+a+b+\alpha)}
\end{aligned}
$$


Example 2.16.

Suppose

$$
F\left(s_{1}, s_{2}, s_{3}\right)=\frac{k}{\left\{a\left(s_{1}+s_{2}\right)^{2}+b\left(s_{1}+s_{2}\right)+c\right\}\left(s_{3}+\alpha\right)\left(s_{3}^{2}-\beta^{2}\right)}
$$

Then, we obtain

$$
\begin{aligned}
G(s)= & \frac{k}{2\left(\beta^{2}-\alpha^{2}\right)}\left[-\frac{2}{a(s+\alpha)^{2}+b(s+\alpha)+c}+\frac{\beta-\alpha}{\beta\left\{a(s-\beta)^{2}+b(s-\beta)+c\right\}}\right. \\
& \left.+\frac{\beta+\alpha}{\beta\left\{a(s+\beta)^{2}+b(s+\beta)+c\right\}}\right] .
\end{aligned}
$$

\section{CONCLUSIONS.}

Theorems on associated transforms developed in this paper are rigorous and should be very useful in calculating the inverse Laplace transform for certain functions. These results should be applicable for obtaining solutions of a wide class of nonlinear equations, which may be encountered frequently in systems engineering. Moreover, these theorems can directly be applied to derive many new associated pairs, and thus one can easily extend the tables given in [5-7] many fold. The results of this paper will help develop more basic theorems in this direction and will apear in subsequent papers.

\section{ACKNOWLEDGEMENT.}

Authors express their grateful thanks to the University of Wisconsin at River Falls for providing financial support in publishing this paper.

\section{REFERENCES}

[1] Volterra, V., Theory of Functionals and of Integral and Integro-differential Equations, Blackie \& Sons, London, 1930.

[2] Wiener, N., Response of a Non-linear Device to Noise, Report 129, Radiation Laboratory, M.I.T., 1942.

[3] Brilliant, M. B., Theory of the Analysis of Nonlinear Systems, Report 345, Research Laboratory of Electronics, M.I.T., 1958.

[4] Barrett, J. F., The Use of Functionals in the Analysis of Nonlinear Physical Systems, J. Electron. Control, Vol. 15, pp. 567-615, 1963.

[5] Lubbock, J. K. and Bansal, V.S., Multidimensional Laplace Transforms for Solution of Nonlinear Equations, Proc. IEE, Vol. 116, NO. 12, December 1969, pp. 2075-2082.

[6] Chen, C. F. and Chiu, R. F., New Theorems of Association of Variables in Multiple Dimensional Laplace Transform, Int. J. Systems Sci, Vol. 4, No. 4, 1973, pp.
647-660.

[7] Koh, E. L., Association of variables in n-dimensional Laplce Transform, Int. J. Systems Sci., Vol. 6, No. 2, 1975, pp. 127-131.

[8] Robert, G. E. and Kaufman, H., Table of Laplace Transforms, W. B. Saunders Co., London, 1966.

\footnotetext{
* Present address of authors: Department of Mathematics and Computer Science, Winona State University, Winona, MN
} 


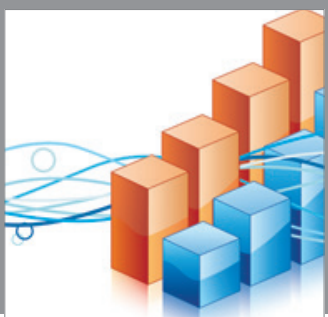

Advances in

Operations Research

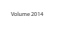

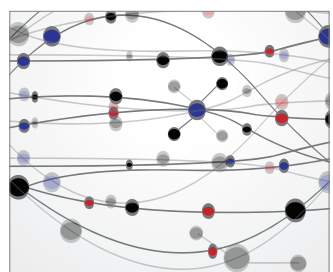

\section{The Scientific} World Journal
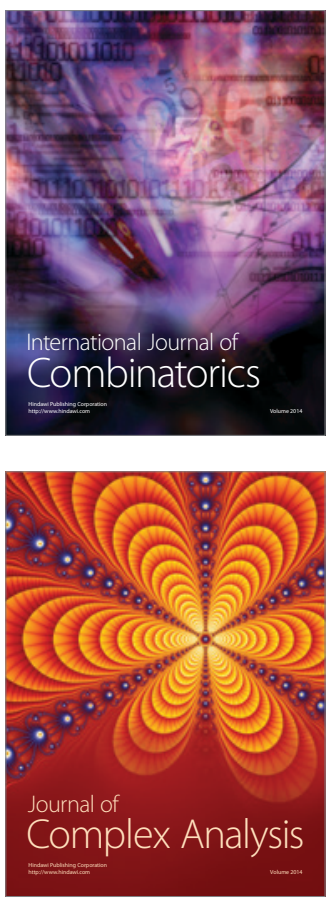

International Journal of

Mathematics and

Mathematical

Sciences
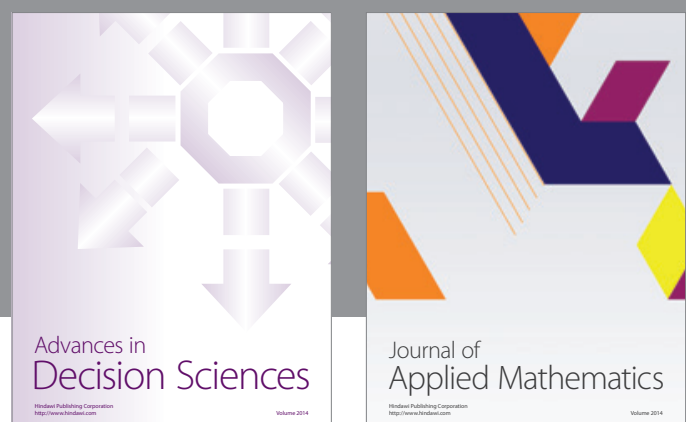

Journal of

Applied Mathematics
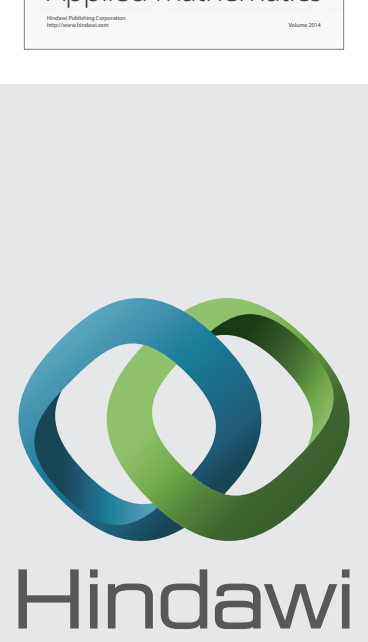

Submit your manuscripts at http://www.hindawi.com
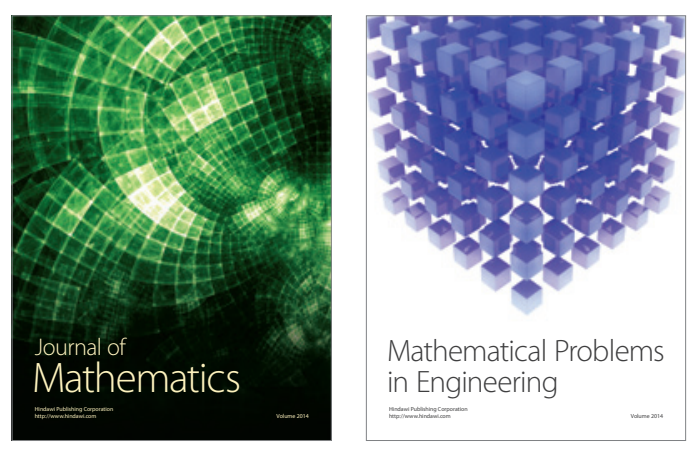

Mathematical Problems in Engineering
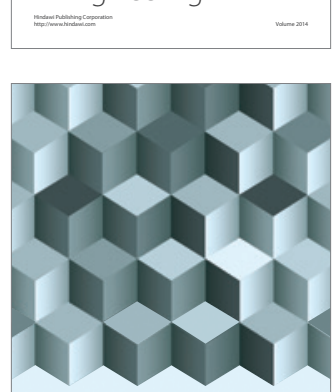

Journal of

Function Spaces
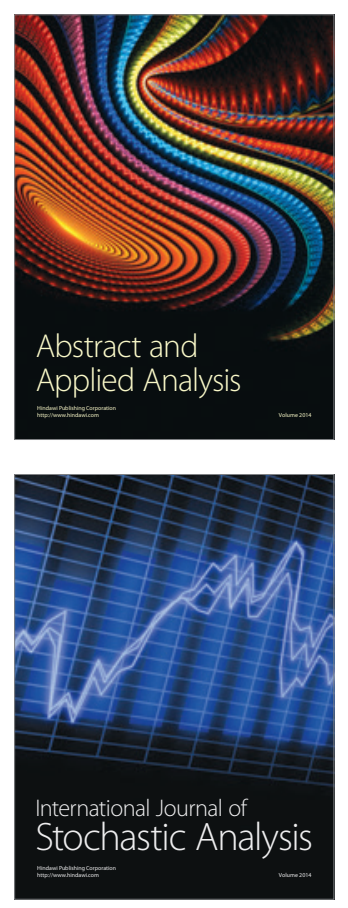

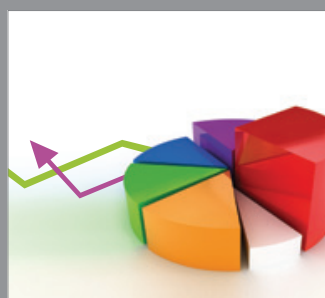

ournal of

Probability and Statistics

Promensencen
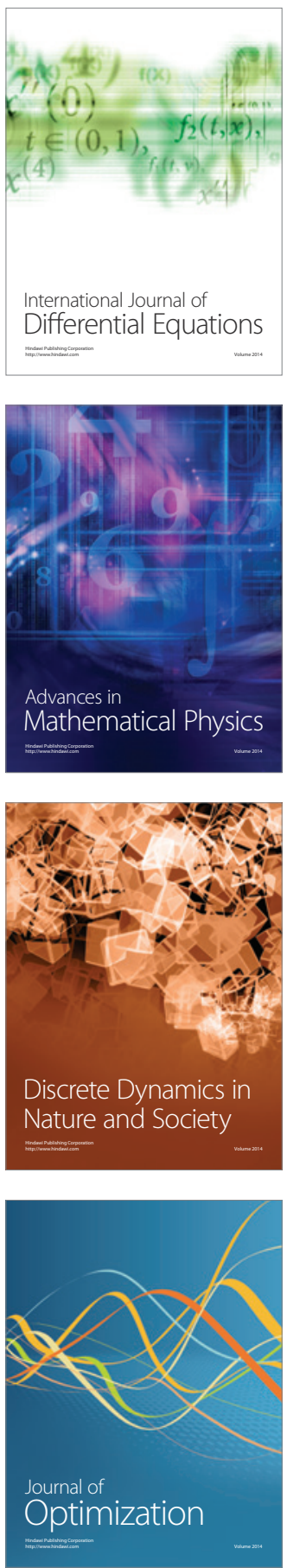